\title{
Synthesis and In Vitro Antibacterial Activity of Catechol-spiramycin Conjugates
}

\author{
Hervé Poras and Gerhard KunesCh* \\ Laboratoire de Chimie Bioorganique et Bioinorganique (CNRS, URA 1384), \\ Université de Paris-Sud, Centre d'Orsay, \\ F-91405 Orsay CEDEX, France \\ Jean-Claude Barrière and Nadine Berthaud \\ Centre de Recherches Rhône-Poulenc Rorer, \\ 13, quai Jules Guesde, B.P. 14, \\ F-94403 Vitry-sur-Seine CEDEX, France
}

ANTOINE ANDREMONT

Service de Microbiologie, CHU Bichat-Claude Bernard, 46, rue Henri Huchard, F-75877 Paris CEDEX, France

(Received for publication May 19, 1998)

The first synthesis of siderophore conjugates of two macrolide antibiotics, spiramycin 1 and neospiramycin 2, which are unable to penetrate the outer membrane of Gramnegative bacteria are described. These novel conjugates were prepared by regioselective acylation of a hydroxyl function of $\mathbf{1}$ and $\mathbf{2}$ with a dihydroxybenzoic Fe(III) complexing ligand linked via a carboxyl group containing spacer to the macrolide antibiotics. The preliminary biological evaluation of these novel conjugates under standard and iron depleted conditions has shown that their antibacterial activity was comparable to that of spiramycin $\mathbf{1}$ and neospiramycin $\mathbf{2}$.

Infections due to various bacteria and viruses are a major cause of mortality worldwide. It has been demonstrated that some of the bacteria involved in these infections produce iron(III) complexing compounds of low molecular weight called siderophores, which contribute to the virulence by depriving the host of iron ${ }^{1}$.

Bacteria are gradually becoming more and more resistant to antibacterial agents. Thus antibacterials with increased activity are constantly needed. Antibiotics such as macrolides are unable to penetrate the outer membrane of Gram-negative bacteria while being active on the ribosomal target.

Recently, several groups have demonstrated that the adjunction of iron complexing ligands to $\beta$-lactam antibiotics greatly improves their biological activity against Gram-negative bacteria ${ }^{2,3)}$. This astute application was inspired by the existence of a few natural compounds like the ferrimycins ${ }^{4)}$ and the albomycins ${ }^{5)}$ which combine strong iron chelators with an antibiotic moiety within the same molecular assembly.

These results and our own interest in this field ${ }^{6)}$ encouraged us to apply this approach (the "Trojan horse

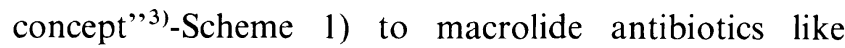
spiramycin 1 and neospiramycin 2.

\section{Chemistry}

Spiramycin 1 is a natural 16-membered macrolide produced by Streptomyces ambofaciens ${ }^{7}$. While the hydrogenation of the two conjugated double bonds does not seriously affect the biological activity of $\mathbf{1}$ and $\mathbf{2}^{\mathbf{8})}$, the aldehyde function and the presence of the aminosugars mycaminose and forosamine are essential for their antibacterial activity (Scheme 2).

Previous studies have shown that the acylation of $\mathbf{1}$ at position 4" does not alter significantly its activity against bacteria $^{8)}$. Compound 1 possesses four hydroxyl groups and their reactivity towards esterification decreases in the order $2^{\prime}, 4^{\prime \prime}, 3$ to $3^{\prime \prime}$. Taking these facts into account, 
Scheme 1.

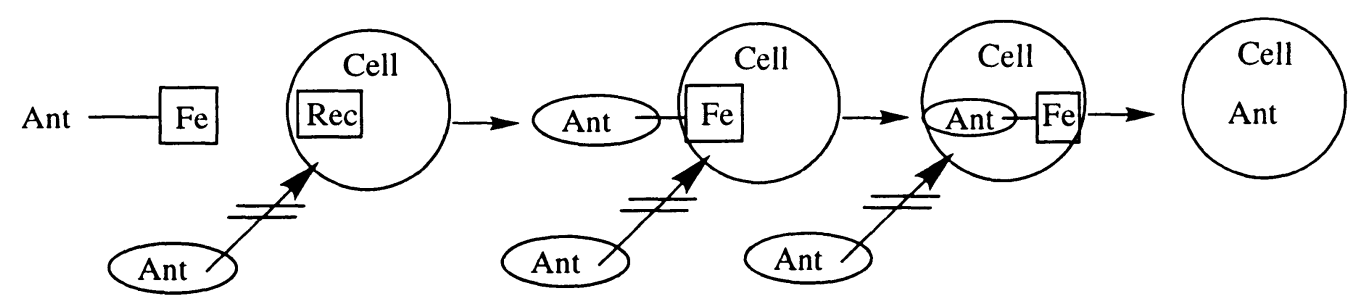

Trojan horse concept. Ant: antibiotic; Rec; Fe-transport receptor.

Scheme 2.

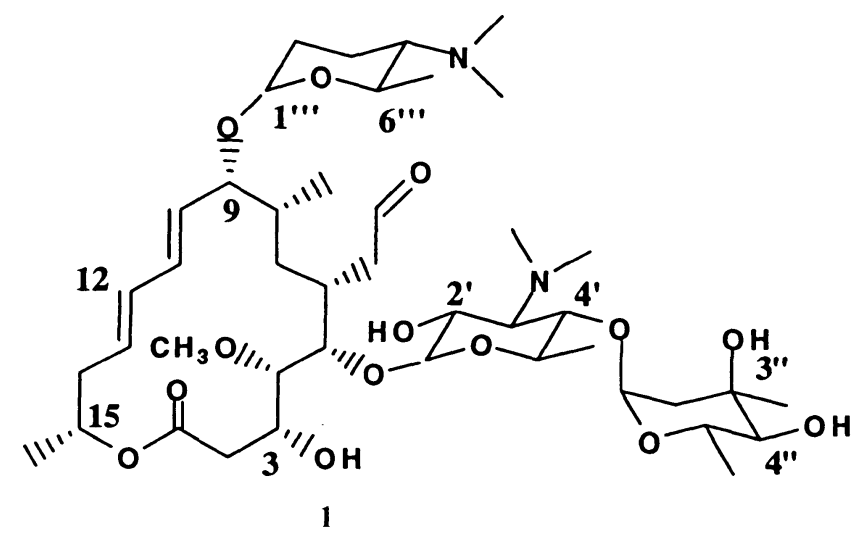

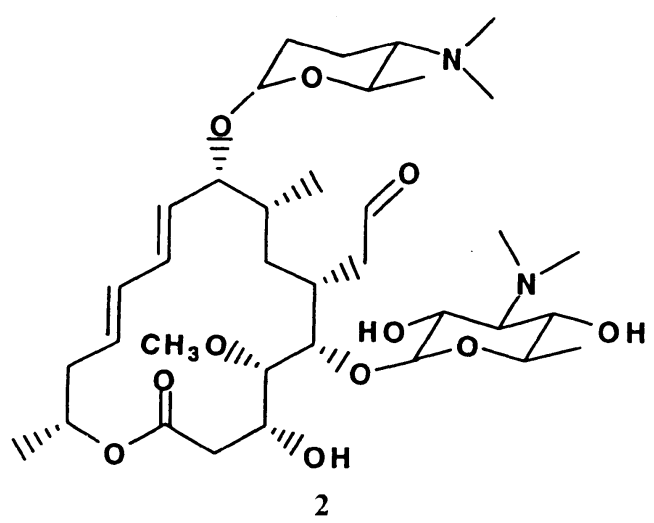

Scheme 3.

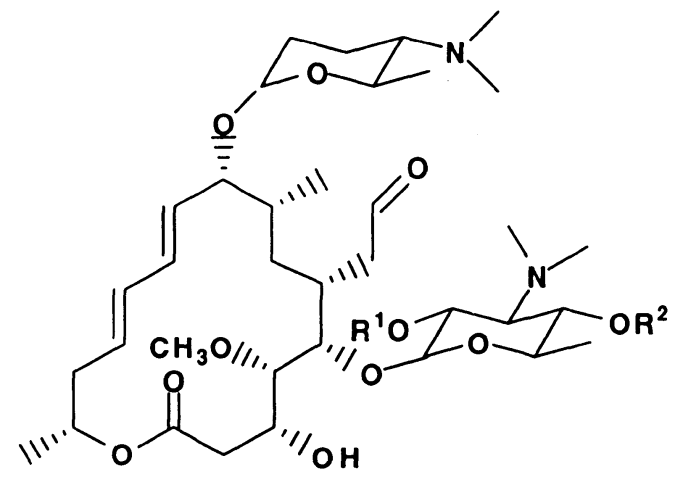

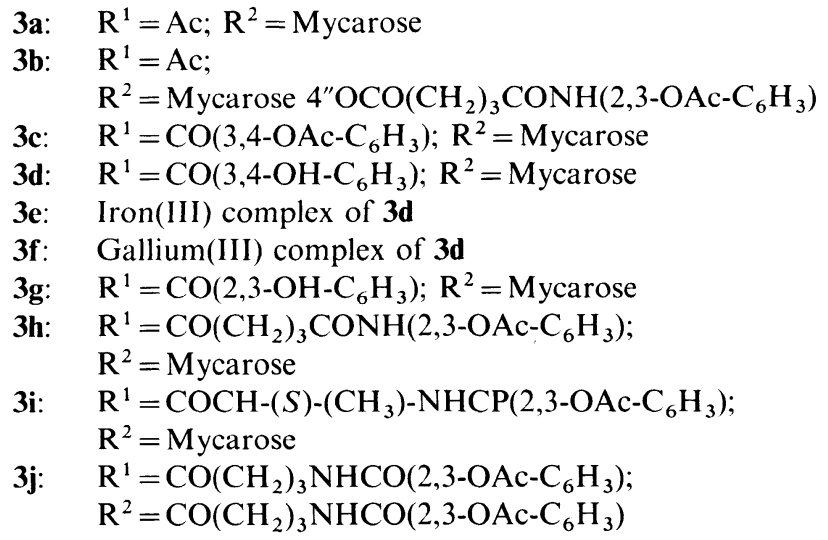

acylation at position $2^{\prime}$ providing the intermediate $\mathbf{3 a}$ turned out to be the method of choice to protect the $2^{\prime}$ position. 4"-O-Acylation of $\mathbf{3 a}$ followed by treatment of the resulting $2^{\prime}-O$-acetyl-4"-O-acyl derivative with guanidine $^{9)}$ furnished $2^{\prime}$-hydroxyl-4"-O-acyl derivatives under mild conditions.

Catechols and hydroxamates are the prevalent ligands among natural bacterial siderophores. They generally involve 3 divalent complexing sites in order to allow for the octahedral geometry around the Fe(III) cation. Our earlier attemps to synthesize conjugates able to transport antibiotics via the active iron transport system provided interesting results with some mono- and dicatechol adducts of pristinamycin $\mathrm{I}_{\mathrm{A}}{ }^{6)}$.

Protection of the catechol unit turned out to be a major and crucial problem for the success of our investigations. Methylation of the phenolic hydroxyl groups was excluded since their cleavage with $\mathrm{BBr}_{3}$ 
Scheme 4.
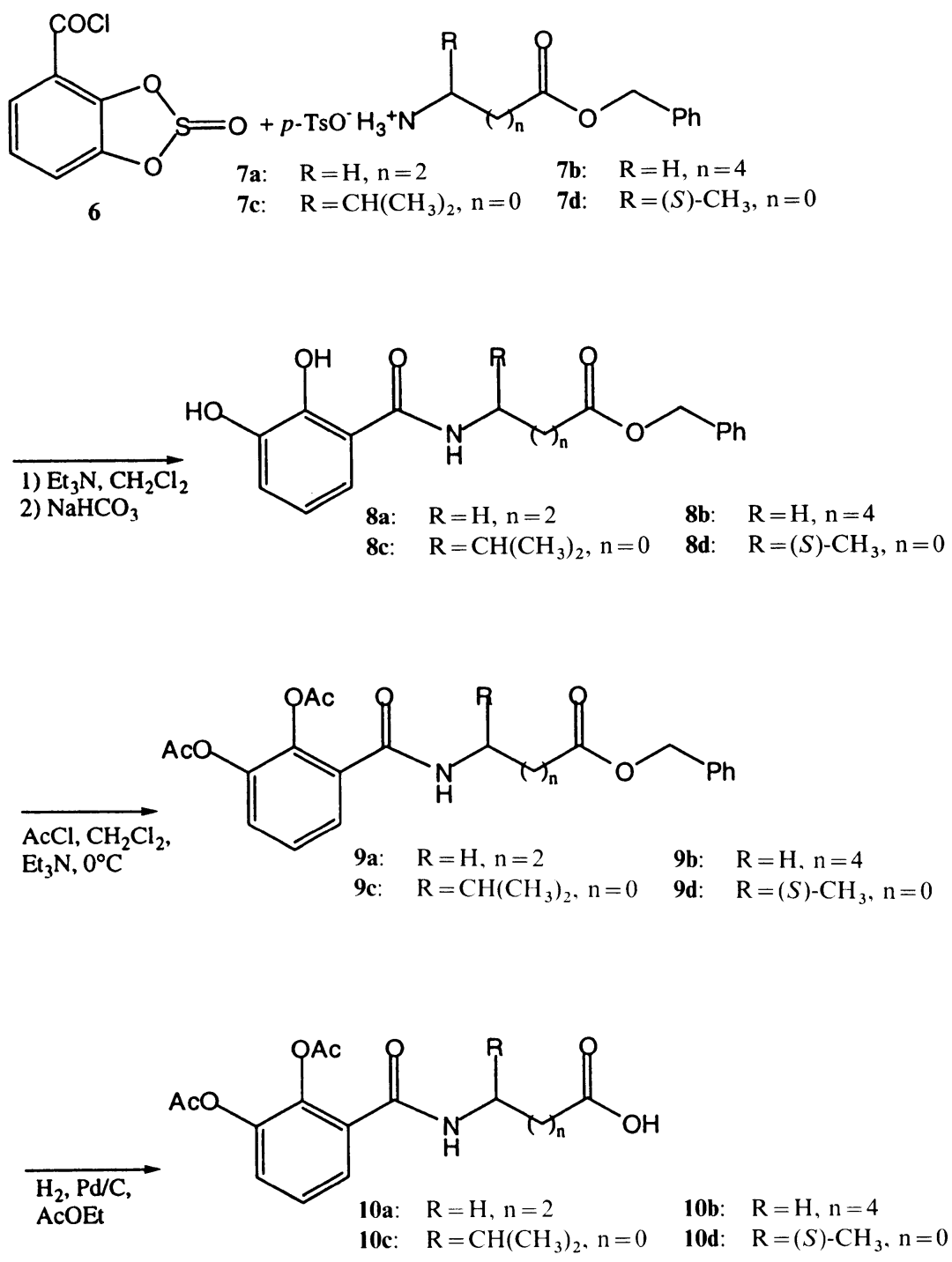

destroys inevitably the spiramycin moiety, as well as hydrogenolysis of benzyl protected derivatives due to the presence of the double bonds in the macrolide ring. On the other hand, although phenolic acetates are rapidly hydrolyzed in the presence of bases, particularly amines, necessitating a careful choice of reaction conditions, they possess the decisive advantage of being easily hydrolyzed under biological conditions as demonstrated by $\mathbf{R}$. REISSBRODT et al. ${ }^{10)}$.

We chose various natural amino acids as spacers to link an hydroxyl group of the antibiotic to a dihydroxybenzoic acid for two major reasons: the eventual control of the release by enzymatic hydrolysis in vivo of the spacer and the absence of any forseeable toxicity of their metabolites.

Thus, several amino acids were protected as benzyl esters $7 \mathbf{a} \sim 7 \mathbf{d}$ by heating them in a solution of benzyl alcohol and $p$-toluene sulfonic acid in toluene. Reaction with 2,3-dioxosulfinyl benzoyl chloride $6^{11)}$ (which can be easily prepared from dihydroxybenzoic acid and thionylchloride) gave compounds 8a $\sim 8 d$ (Scheme 4). Acetylation then deprotection by catalytic hydrogenation provided the building blocks (ligand + spacer) 10a $\sim 10 d$. Finally, the introduction of the ligand moieties 10a $\sim 10 \mathbf{b}$ was realized with classical coupling reagents (DCC or CDI) using either 2 -acetyl-spiramycin $3 \mathbf{a}$ to give the $4^{\prime \prime}$ - $O$-acylated derivative $3 \mathbf{b}$, or on spiramycin 1 leading to 2 '-acylated compounds $\mathbf{3 c}, \mathbf{3 g}, \mathbf{3 h}, \mathbf{3 i}$. Reaction of neospiramycin 2 with 10c under the same conditions furnished a 2',4"-diacylated derivative $3 \mathbf{j}$. The major difficulty encountered with these products was their final purification: chromatography over silicagel gave extremely poor yields of recovered material $(<3 \%)$. Fortunately MPLC over reversed phase (Lichroprep 
RP-18) using a slightly acidic solvent $(\mathrm{pH}=3)$ as eluent allowed the purification of the desired adducts.

The $\mathrm{Fe}(\mathrm{III})$ and $\mathrm{Ga}(\mathrm{III})$ complexes $3 \mathrm{e}$ and $3 \mathrm{f}$ were synthesized from $3 \mathbf{d}$ by treatment with either $\mathrm{Fe}(\mathrm{acac})_{3}$ or gallium nitrate in the presence of triethylamine followed by purification over Sephadex LH-20.

\section{Antibacterial Activity}

The MICs were determined by the standard agar dilution method. Two-fold dilutions of antibiotic in sterile distilled water were prepared from stock solutions and incorporated into melted Mueller-Hinton agar supplemented with $25 \mathrm{mg} /$ liter $\mathrm{Mg}^{++}$and $50 \mathrm{mg} /$ liter $\mathrm{Ca}^{++}$. A Denley Multipoint inoculator was used to apply spots of $1 \mu \mathrm{l}\left(10^{4}\right.$ bacteria) of each bacterial strain studied to plates. Inoculated plates were incubated for 18 hours at $37^{\circ} \mathrm{C}$. For iron depleted conditions, the seed medium consisted of $6 \mathrm{~g}$ of $\mathrm{K}_{2} \mathrm{HPO}_{4}$ (Merck 5101), $3 \mathrm{~g}$ of $\mathrm{KH}_{2} \mathrm{PO}_{4}$ (Merck 4873), $0.2 \mathrm{~g}$ of $\mathrm{MgSO}_{4} \cdot 7 \mathrm{H}_{2} \mathrm{O}$
(Prolabo 25165.292), $1 \mathrm{~g}$ of $\left(\mathrm{NH}_{4}\right)_{2} \mathrm{SO}_{4} \cdot 7 \mathrm{H}_{2} \mathrm{O}$ (Prolabo 21333.296), $4 \mathrm{~g}$ of succinic acid (Merck 682) and $15 \mathrm{~g}$ of agar (Difco). The $\mathrm{pH}$ was adjusted to 7.0 by addition of concentrated sodium hydroxyde (Prolabo 28252.293).

Several Gram-negative strains like Pseudomonas aeruginosa Z61, Escherichia coli D22 and Salmonella typhimurium TA 1535 possess a permeable outer membrane. This feature allowed us to check wether our new adducts were still capable of binding to the ribosomes of the bacterial cells after the passage through the outer membrane. As a matter of course, spiramycin 1 was chosen as the reference compound.

Under Mueller-Hinton conditions (Table 1), the siderophore receptor proteins are not expressed and no active transport is possible. Spiramycin $\mathbf{1}$ and the novel adducts $\mathbf{3 b}$ to $\mathbf{3 j}$ are only active on the strains possessing a permeable outer membrane and show similar MICs with the exception of compound $\mathbf{3 b}$, which is only active against Salmonella typhimurium TA 1535. It is interesting

Table 1. MIC (mg/liter) of $\mathbf{1}$ and the adducts $\mathbf{3}$ on different strains under Mueller-Hinton growth conditions.

\begin{tabular}{|c|c|c|c|c|c|c|c|c|c|}
\hline & 1 & 3b & $3 \mathbf{c}$ & 3d & $3 \mathbf{e}$ & $3 \mathrm{~g}$ & $3 \mathbf{h}$ & $3 \mathbf{i}$ & $3 \mathbf{j}$ \\
\hline Staphylococcus aureus ATCC25923 & 8 & 32 & $>128$ & 128 & 128 & 64 & 16 & 16 & 64 \\
\hline Staphylococcus aureus 209P & 4 & 16 & 128 & 64 & 64 & 32 & 8 & 4 & 64 \\
\hline Pseudomonas aeruginosa ATCC27853 & $>128$ & $>128$ & $>128$ & $>128$ & $>128$ & $>128$ & $>128$ & $>128$ & $>128$ \\
\hline Pseudomonas aeruginosa ${\mathrm{Z} 61^{\mathrm{a}}}^{\mathrm{a}}$ & 16 & 128 & 128 & $>128$ & $>128$ & 128 & 32 & 16 & 64 \\
\hline Escherichia coli NIHJ-JC2 & $>128$ & $>128$ & $>128$ & $>128$ & $>128$ & $>128$ & $>128$ & $>128$ & $>128$ \\
\hline Escherichia coli $\mathrm{D} 22^{\mathrm{a}}$ & 4 & $>128$ & 64 & 32 & 32 & 16 & 2 & 4 & 32 \\
\hline Salmonella typhimurium IPL & $>128$ & $>128$ & $>128$ & $>128$ & $>128$ & $>128$ & $>128$ & $>128$ & $>128$ \\
\hline Salmonella typhimurium TA $1535^{\mathrm{a}}$ & 8 & 16 & 64 & 64 & 64 & 32 & 4 & 4 & 64 \\
\hline
\end{tabular}

a Gram-negative strain possessing a permeable outer membrane.

Table 2. MIC (mg/liter) of $\mathbf{1}$ and the adducts $\mathbf{3}$ on different strains under iron depressed conditions.

\begin{tabular}{|c|c|c|c|c|c|c|c|c|c|}
\hline & 1 & 3b & $3 \mathbf{c}$ & 3d & $3 \mathbf{e}$ & $3 g$ & $3 \mathbf{h}$ & $3 \mathbf{i}$ & $3 \mathbf{j}$ \\
\hline Staphylococcus aureus ATCC25923 & - & - & - & - & - & - & - & - & - \\
\hline Staphylococcus aureus 209P & - & - & - & - & - & - & - & - & - \\
\hline Pseudomonas aeruginosa ATCC 27853 & $>128$ & $>128$ & $>128$ & $>128$ & $>128$ & $>128$ & $>128$ & $>128$ & $>128$ \\
\hline Pseudomonas aeruginosa ${\mathrm{Z} 61^{\mathrm{a}}}^{\mathrm{a}}$ & 16 & 32 & 64 & 128 & 64 & 32 & 8 & 16 & 32 \\
\hline Escherichia coli NIHJ-JC2 & $>128$ & $>128$ & $>128$ & $>128$ & $>128$ & $>128$ & 128 & $>128$ & $>128$ \\
\hline Escherichia coli $\mathrm{D} 22^{\mathrm{a}}$ & - & - & - & - & - & - & - & - & - \\
\hline Escherichia coli ATCC25922 & 64 & $>128$ & $>128$ & $>128$ & 128 & 128 & 64 & 128 & $>128$ \\
\hline Escherichia coli $\mathrm{N} 51$ & 32 & 64 & 128 & $>128$ & 128 & 128 & 32 & 32 & $>128$ \\
\hline Escherichia coli 642 & $>128$ & $>128$ & $>128$ & $>128$ & $>128$ & $>128$ & 64 & 64 & $>128$ \\
\hline Escherichia coli 722 & 128 & 128 & $>128$ & $>128$ & $>128$ & 128 & 64 & 64 & $>128$ \\
\hline Escherichia coli 823 & 64 & 128 & $>128$ & $>128$ & $>128$ & 128 & 64 & 128 & $>128$ \\
\hline Salmonella typhimurium IPL & 128 & 128 & $>128$ & $>128$ & $>128$ & 128 & 64 & 64 & $>128$ \\
\hline Salmonella typhimurium TA $1535^{\mathrm{a}}$ & - & - & - & - & - & - & - & - & - \\
\hline
\end{tabular}

a Gram-negative strain possessing permeable outer membrane. - : no bacterial growth. 
to note that compounds $\mathbf{3 h}$ and $\mathbf{3 i}$ which are acylated in position $2^{\prime}$ of the mycarose unit of spiramycin with the spacer-ligand units 10a and 10d, show similar MICs when compared to the parent compound $\mathbf{1}$.

Under ferric stress conditions, when bacterial growth was allowed (Table 2), no significiant decrease of the MICs was observed. A few hypotheses can be proposed in order to explain these results:

The adducts may be enzymatically hydrolyzed before reaching their target within the bacterial cell.

The receptor site at the surface of the bacterial cell wall may not recognize mono- or dicatechols.

Finally, even if these "Trojan horses" are recognized by the receptors, their mere size (MW close to 3000, see Exp. Part) may not allow them to pass through the membrane channels.

The synthesis of tricatechol and trihydroxamate conjugates of spiramycin 1 of lower molecular weight, less prone to enzymatic hydrolysis by introducing steric hindrance around the ester linkage, is in due course in our laboratory.

\section{Experimental}

\section{NMR}

To characterize our adducts $\mathbf{3}$, all the hydrogen and carbon atoms of spiramycin were attributed using 2D NMR spectroscopy and their chemical shifts were in agreement with published chemical shifts ${ }^{12)}$. Therefore, only characteristic and significant chemical shifts are given in this section.

NMR spectra were recorded on BRUKER instruments: AM 200, AM 250 or AM 400 at respectively $200 \mathrm{MHz}, 250 \mathrm{MHz}$ or $400 \mathrm{MHz}$ for ${ }^{1} \mathrm{H}$ and $50 \mathrm{MHz}$, $62 \mathrm{MHz}$ or $100 \mathrm{MHz}$ for ${ }^{13} \mathrm{C}$ with deuterated chloroform as internal reference $\left(7.24 \mathrm{ppm}\right.$ for ${ }^{1} \mathrm{H}$ and $77 \mathrm{ppm}$ for $\left.{ }^{13} \mathrm{C}\right)$.

\section{MS}

For compounds with a molecular weight under 700 mass units, a Riber-Mag R-10-10 apparatus was used in the direct introduction mode with chemical ionization by $\mathrm{NH}_{3}$. The higher molecular weights were analyzed by ES-MS or FAB-MS on a Platform (VG Fisons) quadrupole instrument. Calibration was performed using a cesium iodide solution $(10 \mathrm{ng} / \mathrm{ml})$ for the weight range of $250 \sim 1400$ with a precision of 0.5 mass units. The samples were dissolved in methanol and diluted in $0.2 \%$ formic acid solution $(\mathrm{pH}=3)$ /acetonitrile $(50: 50)$ with a concentration of 20 to $100 \mathrm{ng} / \mathrm{ml}$.

\section{HPLC}

HPLC analyses were performed on a Perkin Elmer apparatus equipped with a biocompatible LC250 binary pump and an ultraviolet diode LC 235C. The analytical

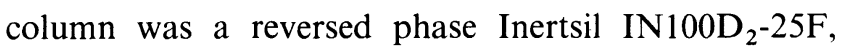
$250 \mathrm{~mm}$ length and $4.6 \mathrm{~mm}$ internal diameter.

\section{MPLC}

$\overline{\text { MPLC }}$ was effected using SDS Chromagel $40 \sim 60 \mu \mathrm{m}$ (230 400 mesh) silicagel or $40 \sim 60 \mu \mathrm{m}$ LiChroprep RP-18 (Merck).

THF and ether were freshly distilled over benzophenone-sodium just before use. Toluene was distilled over $\mathrm{Na}$, pyridine over $\mathrm{KOH}$ and methylene chloride over $\mathrm{P}_{2} \mathrm{O}_{5}$. All the manipulations were effected under argon atmosphere.

\section{2,3-Diacetoxybenzoic Acid 5}

A suspension of 2,3-dihydroxybenzoic acid (5 g; 32.5 $\mathrm{mmol}$ ) in $7 \mathrm{ml}$ of acetic anhydride was stirred at room temperature and two drops of concentrated sulfuric acid were added. After five minutes, a precipitate formed, $35 \mathrm{ml}$ of anhydrous ether were added and the solution was stirred for 12 hours at room temperature. Extraction with $\mathrm{CH}_{2} \mathrm{Cl}_{2}$, washing with cold water, drying over $\mathrm{MgSO}_{4}$ and evaporation of the solvent yielded $7.04 \mathrm{~g}$ of the title compound (91\%).

$\mathrm{C}_{11} \mathrm{H}_{10} \mathrm{O}_{6} \mathrm{MW} 238.20 ;{ }^{1} \mathrm{H}$ NMR $\left(200 \mathrm{MHz}, \mathrm{CDCl}_{3}\right)$ $\delta 2.3(6 \mathrm{H}, \mathrm{s}), 7.2 \sim 7.5(2 \mathrm{H}, \mathrm{m}) 7.9(1 \mathrm{H}, \mathrm{dd}) ; \mathrm{DIC} / \mathrm{NH}_{3}-$ MS $m / z 256(\mathrm{M}+18)^{+\cdot}, m / z 239(\mathrm{M}+\mathrm{H})^{+\cdot} ; \mathrm{mp} 158^{\circ} \mathrm{C}$.

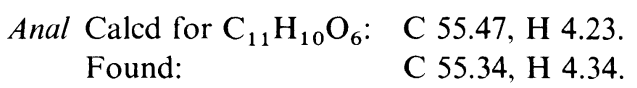

\section{2,3-Dioxosulfinyl Benzoyl Chloride 6}

$1.54 \mathrm{~g}$ of 2,3-dihydroxybenzoic acid $(5 \mathrm{mmol})$ was heated at $40^{\circ} \mathrm{C}$ for 3 hours in $5 \mathrm{ml}$ of $\mathrm{SOCl}_{2}$ with a catalytic amount of urea. The reaction mixture was evaporated several times with toluene at a temperature below $55^{\circ} \mathrm{C}$ to yield $1.06 \mathrm{~g}$ of the title compound $(97 \%)$.

$\mathrm{C}_{7} \mathrm{H}_{3} \mathrm{O}_{4} \mathrm{SCl} \mathrm{MW} 218.61 ;{ }^{1} \mathrm{H}$ NMR $\left(200 \mathrm{MHz}, \mathrm{CDCl}_{3}\right)$ $\delta 7.3(1 \mathrm{H}, \mathrm{t}), 7.5(1 \mathrm{H}, \mathrm{d}), 7.9(1 \mathrm{H}, \mathrm{d}) ;$ IE-MS $m / z 219$ $(\mathrm{M}+2)^{+}, m / z=218(\mathrm{M})^{+} ; \operatorname{mp~} 85^{\circ} \mathrm{C}$.

Anal Calcd for $\mathrm{C}_{7} \mathrm{H}_{3} \mathrm{O}_{4} \mathrm{SCl}$ :

C $38.46, \mathrm{H} 1.38, \mathrm{~S} 14.67, \mathrm{Cl} 16.22$.

Found: C 39.41, H 1.51, S 13.83, Cl 16.15.

\section{$\omega$-Aminobenzyl Esters 7}

$1.03 \mathrm{~g}$ ( $100 \mathrm{mmol}) \mathrm{GABA}, 19.4 \mathrm{~g}$ (1.02 equiv) of $p$ toluene sulfonic acid and $40 \mathrm{ml}$ of benzyl alcohol were heated for 1 hour under reflux in $20 \mathrm{ml}$ of toluene using a Dean-Stark apparatus. After cooling, $200 \mathrm{ml}$ of freshly 
distilled ether were added and the title compound $7 \mathbf{a}$ precipitated at low temperature (yield $84 \%$ ). Compounds 7b, 7c and 7d were prepared using the same procedure.

7a: $\mathrm{C}_{18} \mathrm{H}_{23} \mathrm{NO}_{5} \mathrm{~S}$, MW 365.44; Yield 84\%; (DIC/ $\mathrm{NH}_{3}$ )-MS $m / z 204(\mathrm{M}+\mathrm{H})^{+\cdot}$ (amino compound).

7b: $\mathrm{C}_{20} \mathrm{H}_{27} \mathrm{NO}_{5} \mathrm{~S}$, MW 393.50; Yield 85\%; (DIC/ $\mathrm{NH}_{3}$ )-MS $m / z 222(\mathrm{M}+\mathrm{H})^{+\cdot}$ (amino compound).

7c: $\mathrm{C}_{19} \mathrm{H}_{25} \mathrm{NO}_{5} \mathrm{~S}$, MW 379.47; Yield 60\%; (DIC/ $\mathrm{NH}_{3}$ )-MS $m / z 216(\mathrm{M}+\mathrm{H})^{+\cdot}$ (amino compound).

7d: $\mathrm{C}_{17} \mathrm{H}_{21} \mathrm{NO}_{5} \mathrm{~S}$, MW 351.42; Yield 68\%; (DIC/ $\mathrm{NH}_{3}$ )-MS $m / z 188(\mathrm{M}+\mathrm{H})^{+\cdot}$ (amino compound).

\section{$\mathrm{N}$-(2,3-Dihydroxybenzoyl)aminobenzylic Esters 8}

To a stirred, cooled solution of $1.38 \mathrm{~g}(6.33 \mathrm{mmol})$ 2,3-dioxosulfinyl benzoyl chloride 6 ( 1.2 equiv) and $3.73 \mathrm{~g}$ of $p$-toluene sulfonate benzylic ester $7 \mathbf{a}(9.5 \mathrm{mmol})$ in $100 \mathrm{ml}$ of $\mathrm{CH}_{2} \mathrm{Cl}_{2}, 1.5 \mathrm{ml}$ of $\mathrm{Et}_{3} \mathrm{~N}$ (1.5 equiv) in $10 \mathrm{ml}$ $\mathrm{CH}_{2} \mathrm{Cl}_{2}$ was added dropwise. The reaction was followed by TLC and quenched by a saturated solution of $\mathrm{NaHCO}_{3}$, extracted with $\mathrm{CH}_{2} \mathrm{Cl}_{2}$, dried, evaporated and chromatographed by MPLC $\left(\mathrm{CH}_{2} \mathrm{Cl}_{2} / \mathrm{Et}_{2} \mathrm{O} 5 \%\right)$ to give the title compound $\mathbf{8 a}$. Products $\mathbf{8 b}, \mathbf{8 c}$ and $\mathbf{8 d}$ were prepared using the same procedure.

8a: $\mathrm{C}_{18} \mathrm{H}_{19} \mathrm{NO}_{5}$, MW 329.35; Yield 62\%; ${ }^{1} \mathrm{H}$ NMR $\left(200 \mathrm{MHz}, \mathrm{CDCl}_{3}\right) \delta 2.0(2 \mathrm{H}, \mathrm{m}), 2.5(2 \mathrm{H}, \mathrm{t}) 3.5(2 \mathrm{H}$, q), $5.1(2 \mathrm{H}, \mathrm{s}), 6.8(1 \mathrm{H}, \mathrm{t}), 6.91 \mathrm{H}, \mathrm{d}), 7.0(1 \mathrm{H}, \mathrm{d}), 7.4$ $(5 \mathrm{H}, \mathrm{s}) ;\left(\mathrm{DIC} / \mathrm{NH}_{3}\right)-\mathrm{MS} m / z=330(\mathrm{M}+\mathrm{H})^{+\cdot}$.

8b: $\mathrm{C}_{20} \mathrm{H}_{23} \mathrm{NO}_{5}$, MW 357.41; Yield $28 \%$; ${ }^{1} \mathrm{H}$ NMR $\left(250 \mathrm{MHz} \mathrm{CDCl}_{3}\right) \delta 1.3(2 \mathrm{H}, \mathrm{m}), 1.6(4 \mathrm{H}, \mathrm{m}), 2.3(2 \mathrm{H}$, t), $3.4(2 \mathrm{H}, \mathrm{q}), 5.1(2 \mathrm{H}, \mathrm{s}), 6.8(1 \mathrm{H}, \mathrm{t}), 6.9(1 \mathrm{H}, \mathrm{d}), 7.0$ $(1 \mathrm{H}, \mathrm{d}), \quad 7.4 \quad(5 \mathrm{H}, \mathrm{s}) ; \quad\left(\mathrm{DIC} / \mathrm{NH}_{3}\right)-\mathrm{MS} \quad m / z=358$ $(\mathrm{M}+\mathrm{H})^{+\cdot}$.

8c: $\mathrm{C}_{19} \mathrm{H}_{21} \mathrm{NO}_{5}$, MW 343.38; Yield 33\%; ${ }^{1} \mathrm{H}$ NMR $\left(200 \mathrm{MHz}^{\mathrm{CDCl}}\right)_{3} \delta 0.95(3 \mathrm{H}, \mathrm{d}), 1.0(3 \mathrm{H}, \mathrm{d}), 2.3(1 \mathrm{H}$, $\mathrm{m}), 4.75(1 \mathrm{H}, \mathrm{dd}), 5.1(2 \mathrm{H}, \mathrm{s}), 6.8(1 \mathrm{H}, \mathrm{t}), 7.0(1 \mathrm{H}, \mathrm{d})$, $7.1(1 \mathrm{H}, \mathrm{d}), 7.4(5 \mathrm{H}, \mathrm{s}) ;\left(\mathrm{DIC} / \mathrm{NH}_{3}\right)-\mathrm{MS} m / z=344$ $(\mathrm{M}+\mathrm{H})^{+\cdot}$.

8d: $\mathrm{C}_{17} \mathrm{H}_{17} \mathrm{NO}_{5}$, MW 315.33; Yield 67\%; ${ }^{1} \mathrm{H}$ NMR $\left(250 \mathrm{MHz}, \mathrm{CDCl}_{3}\right) \delta 1.5(3 \mathrm{H}, \mathrm{d}), 4.8(1 \mathrm{H}, \mathrm{q}), 5.2(2 \mathrm{H}$, s), $6.8(1 \mathrm{H}, \mathrm{t}), 6.9(1 \mathrm{H}, \mathrm{d}), 7.1(1 \mathrm{H}, \mathrm{d}), 7.4(5 \mathrm{H}, \mathrm{s})$; $\left(\mathrm{DIC} / \mathrm{NH}_{3}\right)-\mathrm{MS} m / z=316(\mathrm{M}+\mathrm{H})^{+}$.

\section{$N$-(2,3-Diacetoxybenzoyl)aminobenzylic Esters 9}

To a stirred solution of $\mathbf{8 a}(1.06 \mathrm{mmol})$ in $60 \mathrm{ml}$ $\mathrm{CH}_{2} \mathrm{Cl}_{2}, 0.5 \mathrm{ml}$ of $\mathrm{Et}_{3} \mathrm{~N}$ was added. Then, the reaction mixture was cooled to $0^{\circ} \mathrm{C}$ and $0.15 \mathrm{ml}$ of acetyl chloride $(2.12 \mathrm{mmol})$ in $5 \mathrm{ml} \mathrm{CH}_{2} \mathrm{Cl}_{2}$ was added dropwise. The reaction mixture was allowed to warm to room temperature and analyzed by TLC. After quenching with water, the organic layer was washed, dried and evap- orated to yield $630 \mathrm{mg}$ of the title compound 9a. Products 9b, 9c and 9d were prepared using the same procedure.

9a: $\mathrm{C}_{22} \mathrm{H}_{23} \mathrm{NO}_{7}$, MW 413.43; Yield 99\%; ${ }^{1} \mathrm{H}$ NMR $\left(200 \mathrm{MHz}, \mathrm{CDCl}_{3}\right) \delta 1.9(2 \mathrm{H}, \mathrm{m}), 2.3(6 \mathrm{H}, \mathrm{s}), 2.4(2 \mathrm{H}$, t), $3.4(2 \mathrm{H}, \mathrm{m}), 5.1(2 \mathrm{H}, \mathrm{s}), 6.4(1 \mathrm{H}, \mathrm{t}), 6.9(2 \mathrm{H}, \mathrm{m}), 7.3$ $(5 \mathrm{H}, \quad \mathrm{s}), \quad 7.4 \quad(1 \mathrm{H}, \mathrm{m}) ; \quad\left(\mathrm{DIC} / \mathrm{NH}_{3}\right)-\mathrm{MS} \quad m / z=414$ $(\mathrm{M}+\mathrm{H})^{+\cdot}$.
Anal Calcd for $\mathrm{C}_{22} \mathrm{H}_{23} \mathrm{NO}_{7}$ : C 63.91, H 5.61, N 3.39. Found:
C 63.03, H 5.45, N 3.49 .

9b: $\mathrm{C}_{24} \mathrm{H}_{27} \mathrm{NO}_{7}, \mathrm{MW} 441.48$; Yield: $91 \%,{ }^{1} \mathrm{H}$ NMR $\left(250 \mathrm{MHz}, \mathrm{CDCl}_{3}\right) \delta 1.35(2 \mathrm{H}, \mathrm{m}), 1.55(2 \mathrm{H}, \mathrm{m}), 1.65$ $(2 \mathrm{H}, \mathrm{m}), 2.25(6 \mathrm{H}, \mathrm{s}), 2.3(2 \mathrm{H}, \mathrm{t}), 3.35(2 \mathrm{H}, \mathrm{q}), 5.1(2 \mathrm{H}$, s), $6.2(1 \mathrm{H}, \mathrm{t}), 7.2(2 \mathrm{H}, \mathrm{m}), 7.3(5 \mathrm{H}, \mathrm{s}), 7.5(1 \mathrm{H}, \mathrm{dd})$; $\left(\mathrm{DIC} / \mathrm{NH}_{3}\right)-\mathrm{MS} m / z=442(\mathrm{M}+\mathrm{H})^{+}$.

$\begin{array}{ll}\text { Anal Calcd for } \mathrm{C}_{24} \mathrm{H}_{27} \mathrm{NO}_{7} \text { : } & \text { C } 65.29, \mathrm{H} 6.16, \mathrm{~N} 3.17 . \\ \begin{array}{ll}\text { Found: } & \text { C } 65.19, \mathrm{H} 6.31, \mathrm{~N} 2.99 .\end{array}\end{array}$

9c: $\mathrm{C}_{23} \mathrm{H}_{25} \mathrm{NO}_{7}, \mathrm{MW} 427.45$; Yield 78\%; ${ }^{1} \mathrm{H}$ NMR $\left(200 \mathrm{MHz}, \mathrm{CDCl}_{3}\right) \delta 0.9(3 \mathrm{H}, \mathrm{d}), 0.95(3 \mathrm{H}, \mathrm{d}), 2.3(1 \mathrm{H}$, $\mathrm{m}), 2.3(3 \mathrm{H}, \mathrm{s}), 2.35(3 \mathrm{H}, \mathrm{s}), 4.8(1 \mathrm{H}, \mathrm{dd}), 5.2(2 \mathrm{H}, \mathrm{s})$, 6.9 (1H, d), $7.3(6 \mathrm{H}, \mathrm{m}), 7.7(1 \mathrm{H}, \mathrm{dd}) ;\left(\mathrm{DIC} / \mathrm{NH}_{3}\right)-\mathrm{MS}$ $m / z=428(\mathrm{M}+\mathrm{H})^{+}$.

$\begin{array}{ll}\text { Anal Calcd for } \mathrm{C}_{23} \mathrm{H}_{25} \mathrm{NO}_{7}: & \mathrm{C} 64.62, \mathrm{H} 5.90, \mathrm{~N} 3.28 . \\ \text { Found: } & \text { C } 62.89, \mathrm{H} 5.57, \mathrm{~N} 3.23 .\end{array}$

9d: $\mathrm{C}_{21} \mathrm{H}_{21} \mathrm{NO}_{7}, \mathrm{MW}$ 399.40; Yield 94\%; ${ }^{1} \mathrm{H}$ NMR $\left(200 \mathrm{MHz} \mathrm{CDCl}_{3}\right) \delta 1.45(3 \mathrm{H}, \mathrm{d}), 2.3(3 \mathrm{H}, \mathrm{s}), 2.4(3 \mathrm{H}$, s), $4.8(1 \mathrm{H}, \mathrm{q}), 5.2(2 \mathrm{H}, \mathrm{s}), 7.3(7 \mathrm{H}, \mathrm{m}), 7.7(1 \mathrm{H}, \mathrm{dd})$; $\left(\mathrm{DIC} / \mathrm{NH}_{3}\right)-\mathrm{MS} m / z=400(\mathrm{M}+\mathrm{H})^{+\cdot}$.

Anal Calcd for $\mathrm{C}_{21} \mathrm{H}_{21} \mathrm{NO}_{7}$ : C 63.15, H 5.30, N 3.51. Found:

C 61.76, H 5.26, N 3.23 .

\section{$\mathrm{N}$-(2,3-Diacetoxybenzoyl) Acids $\mathbf{1 0}$}

$588 \mathrm{mg}(1.33 \mathrm{mmol})$ of benzylic ester 9a was stirred in $10 \mathrm{ml}$ ethyl acetate under atmospherique pressure of $\mathrm{H}_{2}$ with $20 \%$ equiv wt of $10 \% \mathrm{Pd} / \mathrm{C} 10 \%$ (118 mg). The reaction mixture was stirred for 12 hours, then the catalyst was filtered and the solvent was evaporated. Products 10b, 10c and 10d were prepared using the same procedure.

10a: $\mathrm{C}_{15} \mathrm{H}_{17} \mathrm{NO}_{7}$, MW 323.30; Yield 84\%; ${ }^{1} \mathrm{H}$ NMR $\left(200 \mathrm{MHz} \mathrm{CDCl}_{3}\right) \delta 1.9(2 \mathrm{H}, \mathrm{m}), 2.3(6 \mathrm{H}, \mathrm{s}), 2.45(2 \mathrm{H}$, t), $3.4(2 \mathrm{H}, \mathrm{q}), 6.4(1 \mathrm{H}, \mathrm{t}), 7.3(2 \mathrm{H}, \mathrm{m}), 7.4(1 \mathrm{H}, \mathrm{dd})$; ${ }^{13} \mathrm{C} \mathrm{NMR}\left(100 \mathrm{MHz} \mathrm{CDCl}_{3}\right) \delta 20.14,20.26,24.04,30.96$, $38.91,125.45,125.98,126.22,130.00,139.78,142.58$, 165.62, 168.06, 168.19, 177.10; (DIC/NH $)_{3}-\mathrm{MS} m / z 324$ $(\mathrm{M}+\mathrm{H})^{+\cdot}, m / z=341(\mathrm{M}+18)^{+\cdot}$.

Anal Calcd for $\mathrm{C}_{15} \mathrm{H}_{17} \mathrm{NO}_{7}$ : C 63.91, H 5.61, N 3.39 . Found:

C 63.03, H 5.45, N 3.49 .

10b: $\mathrm{C}_{17} \mathrm{H}_{21} \mathrm{NO}_{7}$; MW 351.36; Yield 82\%; ${ }^{1} \mathrm{H}$ NMR $\left(200 \mathrm{MHz} \mathrm{CDCl}_{3}\right) \delta 1.4(2 \mathrm{H}, \mathrm{q}), 1.65(4 \mathrm{H}, \mathrm{m}), 2.4(6 \mathrm{H}$, 
s), $2.4(2 \mathrm{H}, \mathrm{m}), 3.4(2 \mathrm{H} ; \mathrm{q}), 6.2(1 \mathrm{H}, \mathrm{t}), 7.2(2 \mathrm{H}, \mathrm{m}), 7.6$ $(1 \mathrm{H}, \mathrm{dd}) ;{ }^{13} \mathrm{C}$ NMR $\left(100 \mathrm{MHz}, \mathrm{CDCl}_{3}\right) \delta 20.14,20.23$, 23.96, 25.88, 28.35, 33.50, 39.44, 125.32, 125.98, 126.21, $130.23,139.64,142.55,165.28,167.90,168.08,177.93$; $\left(\mathrm{DIC} / \mathrm{NH}_{3}\right)-\mathrm{MS} m / z=338(\mathrm{M}+\mathrm{H})^{+\cdot}, m / z=355(\mathrm{M}+$ $18)^{+}$.

$$
\begin{array}{ll}
\text { Anal Calcd for } \mathrm{C}_{17} \mathrm{H}_{21} \mathrm{NO}_{7} \text { : } & \text { C } 58.10, \mathrm{H} 6.03, \mathrm{~N} 3.99 . \\
\text { Found: } & \text { C } 56.55, \mathrm{H} 6.29, \mathrm{~N} 3.32 .
\end{array}
$$

10c: $\mathrm{C}_{16} \mathrm{H}_{19} \mathrm{NO}_{7}$, MW 337.33; Yield 90\%; ${ }^{1} \mathrm{H}$ NMR $\left(250 \mathrm{MHz}, \mathrm{CDCl}_{3}\right) \delta 1.0(6 \mathrm{H}, \mathrm{m}), 2.20(1 \mathrm{H}, \mathrm{m}), 2.28(3 \mathrm{H}$, s), $2.33(3 \mathrm{H}, \mathrm{s}), 4.77(1 \mathrm{H}, \mathrm{dd}), 6.9(1 \mathrm{H}, \mathrm{d}), 7.3(1 \mathrm{H}, \mathrm{m})$, $7.7(1 \mathrm{H}, \mathrm{m}) ;{ }^{13} \mathrm{C} \mathrm{NMR}\left(100 \mathrm{MHz}, \mathrm{CDCl}_{3}\right) \delta 7.49,18.79$, $20.34,20.50,31.0,57.38,126.27,126.58,127.21,129.14$, 139.88, 142.63, 164.90, 168.05, 168.14, 175.19; (DIC/ $\left.\mathrm{NH}_{3}\right)$-MS $m / z=338(\mathrm{M}+\mathrm{H})^{+}, m / z 355(\mathrm{M}+18)^{+\cdot}$.

Anal Calcd for $\mathrm{C}_{16} \mathrm{H}_{19} \mathrm{NO}_{7}$ : C 56.97, H 5.68, N 4.15. Found:

C 56.78, H 5.73, N 4.09 .

10d: $\mathrm{C}_{14} \mathrm{H}_{15} \mathrm{NO}_{7}$, MW 309.28; Yield 81\%; ${ }^{1} \mathrm{H}$ NMR $\left(200 \mathrm{MHz}, \mathrm{CDCl}_{3}\right) \delta 1.5(3 \mathrm{H}, \mathrm{d}), 2.3(3 \mathrm{H}, \mathrm{s}), 2.4(3 \mathrm{H}$, s), $4.75(1 \mathrm{H}, \mathrm{q}), 7.3(2 \mathrm{H}, \mathrm{m}), 7.7(1 \mathrm{H}, \mathrm{m}) ;{ }^{13} \mathrm{C} \mathrm{NMR}$ $\left(100 \mathrm{MHz}, \mathrm{CDCl}_{3}\right) \delta 17.69,20.08,20.26,48.31,126.16$, $126.37,126.94,128.65,139.82,142.51,164.47,167.88$, 168.10, 175.30; (DIC/NH $)_{3}-\mathrm{MS} m / z=310(\mathrm{M}+\mathrm{H})^{+\cdot}$, $m / z=327(\mathrm{M}+18)^{+}$.

$\begin{array}{ll}\begin{array}{l}\text { Anal Calcd for } \mathrm{C}_{14} \mathrm{H}_{15} \mathrm{NO}_{7}: \\ \text { Found: }\end{array} & \text { C } 54.37, \mathrm{H} 4.89, \mathrm{~N} 4.53 . \\ & \text { C } 54.89, \mathrm{H} 5.61, \mathrm{~N} 2.99 .\end{array}$

\section{2'-O-Acetyl Spiramycin 3a}

The literature procedure ${ }^{12}$ ) yielded $93 \%$ of the title compound 3a. HPLC analysis showed a purity superior to $95 \% . \quad \mathrm{C}_{45} \mathrm{H}_{76} \mathrm{~N}_{2} \mathrm{O}_{15}, \quad \mathrm{MW} 885.11 ;{ }^{1} \mathrm{H}$ NMR $\left(400 \mathrm{MHz}, \mathrm{CDCl}_{3}\right) \delta 2.2\left(3 \mathrm{H}, \mathrm{COCH}_{3}, \mathrm{~s}\right), 4.9\left(1 \mathrm{H}, \mathrm{H}_{2}\right.$, dd); ${ }^{13} \mathrm{C}$ NMR $\delta 205.67\left(\mathrm{COCH}_{3}\right) ;\left(\mathrm{DIC} / \mathrm{NH}_{3}\right)-\mathrm{MS} m / z$ $886(\mathrm{M}+\mathrm{H})^{+\cdot}$, ES-MS $m / z=885.6(\mathrm{M}+\mathrm{H})^{+\cdot}, m / z=$ $741.6(\mathrm{M}-\text { mycarose })^{+}$.

Anal Calcd for $\mathrm{C}_{45} \mathrm{H}_{76} \mathrm{~N}_{2} \mathrm{O}_{15}$ : C 61.07, H 8.65, N 3.16. Found:

C 58.66, H 8.05, N 3.43 .

2'-O-Acetyl-4" $-O-[4-N-(2,3-$ diacetoxybenzoyl) $]$ butanoyl Spiramycin 3b

$238 \mathrm{mg}$ of compound $10 \mathrm{a}(0.73 \mathrm{mmol} ; 1.2$ equiv) were dissolved in $10 \mathrm{ml}$ of $\mathrm{CH}_{2} \mathrm{Cl}_{2}$ at room temperature and $152 \mathrm{mg}$ of DCC ( $0.73 \mathrm{mmol} ; 1.2$ equiv) and catalytic amount of PPY (4-pyrrolidinopyridine) ${ }^{14)}$ were added and stirred for one hour. $544 \mathrm{mg}(0.61 \mathrm{mmol} ; 1$ equiv) of 2 '-O-acetyl spiramycin 3a and the mixture was stirred overnight. The precipitate of dicyclohexylurea was filtered. The filtrate was washed with water and the organic layer was dried over $\mathrm{MgSO}_{4}$. The solvent was evaporated and the crude reaction product was chro- matographed by MPLC on reversed phase LiChroprep RP-18 (Merck) with $\mathrm{H}_{2} \mathrm{O}$ (citric acid $\mathrm{pH}=3,450 \mathrm{mg} /$ liter) $74 \% / \mathrm{CH}_{3} \mathrm{CN} 26 \%$ as elution system. After extraction $145 \mathrm{mg}$ of product $3 \mathrm{a}$ were obtained $(20 \%)$.

$\mathrm{C}_{60} \mathrm{H}_{91} \mathrm{~N}_{3} \mathrm{O}_{21}$, MW 1190.4; ${ }^{1} \mathrm{H}$ NMR $(200 \mathrm{MHz}$, $\left.\mathrm{CDCl}_{3}\right) \delta 4.6\left(1 \mathrm{H}, \mathrm{H}_{4^{\prime \prime}}, \mathrm{d}\right), 4.9\left(1 \mathrm{H}, \mathrm{H}_{2^{\prime}}, \mathrm{dd}\right), 7.55(1 \mathrm{H}$, $\mathrm{m}), 7.7(1 \mathrm{H}, \mathrm{m}) ; 8.0(1 \mathrm{H}, \mathrm{m})$; ES-MS $m / z=1190.5$ $(\mathrm{M}+\mathrm{H})^{+}, \quad m / z=1148.5, \quad m / z=1106.5, \quad m / z=927.5$ (McLafferty rearrangement), $m / z=885.5$ (M-substituent in position $\left.4^{\prime \prime}\right)^{+} ; \mathrm{mp} 74 \sim 76^{\circ} \mathrm{C}$.

Anal Calcd for $\mathrm{C}_{60} \mathrm{H}_{91} \mathrm{~N}_{3} \mathrm{O}_{21}$ : C 60.54, H 7.71, N 3.53. Found:

C $58.41, \mathrm{H} 8.40, \mathrm{~N} 2.38$.

\section{2'-O-(3,4-Diacetoxybenzoyl)spiramycin 3c}

The same procedure as for compound $\mathbf{3 b}$ gave the title compound with $14 \%$ yield. $\mathrm{C}_{54} \mathrm{H}_{82} \mathrm{~N}_{2} \mathrm{O}_{19}$, MW 1063.26; ${ }^{1} \mathrm{H} \mathrm{NMR}\left(400 \mathrm{MHz}, \mathrm{CDCl}_{3}\right) \delta 2.9\left(1 \mathrm{H}, \mathrm{H}_{4^{\prime \prime}}, \mathrm{d}\right), 4.9(1 \mathrm{H}$, $\mathrm{H}_{2^{\prime}}$, dd), $7.3(1 \mathrm{H}, \mathrm{d}), 7.8(1 \mathrm{H}, \mathrm{d}), 7.9(1 \mathrm{H}, \mathrm{dd}) ;{ }^{13} \mathrm{C} \mathrm{NMR}$ $\left(100 \mathrm{MHz}, \mathrm{CDCl}_{3}\right) \delta 167.82,167.58\left(\mathrm{COCH}_{3}\right) ; 162.88$ (COAr); ES-MS $m / z 1063.5(\mathrm{M}+\mathrm{H})^{+\cdot}, m / z 1021.5, m / z$ $979.5, \mathrm{~m} / z$ 843.5 $\left(\mathrm{M} \text { - substituent in position } 2^{\prime}\right)^{+} ; \mathrm{mp:}$ $128 \sim 130^{\circ} \mathrm{C}$

$$
\begin{array}{ll}
\text { Anal Calcd for } \mathrm{C}_{54} \mathrm{H}_{82} \mathrm{~N}_{2} \mathrm{O}_{19} \text { : } & \text { C } 61.0, \mathrm{H} 7.77, \mathrm{~N} 2.63 . \\
\text { Found: } & \text { C 59.24, H 7.44, N 2.74. }
\end{array}
$$

\section{2'-O-(3,4-Dihydroxybenzoyl)spiramycin 3d}

$230 \mathrm{mg}(10 \mathrm{mmol})$ of sodium was added under argon to $8 \mathrm{ml}$ of anhydrous methanol at $0^{\circ} \mathrm{C}$. After dissolving, $960 \mathrm{mg}(10 \mathrm{mmol})$ of guanidine hydrochloride were added and the solution was stirred during a few minutes and the precipitate of $\mathrm{NaCl}$ was filtered. $130 \mathrm{mg}$ of $2^{\prime}-O$ (3,4-diacetoxybenzoyl)spiramycin $3 \mathbf{c}$ were dissolved in $5 \mathrm{ml} \mathrm{EtOH}-\mathrm{CH}_{2} \mathrm{Cl}_{2} 9 / 1.150 \mathrm{ml}$ of the above solution was introduced ( 1 equiv guanidine) and the solution was stirred 15 minutes at room temperature. Then, the solvent was evaporated, the product was dissolved in $\mathrm{CH}_{2} \mathrm{Cl}_{2}$ and washed with water. After drying and evaporation of the solution, $119 \mathrm{mg}$ of compound 3d were obtained $(99 \%) . \mathrm{C}_{50} \mathrm{H}_{78} \mathrm{~N}_{2} \mathrm{O}_{17}, \mathrm{MW} 979.18 ;{ }^{1} \mathrm{H} \mathrm{NMR}(400 \mathrm{MHz}$, $\left.\mathrm{CDCl}_{3}\right) \delta 2.9\left(1 \mathrm{H}, \mathrm{H}_{4^{\prime \prime}}, \mathrm{d}\right), 4.9 \mathrm{ppm}\left(1 \mathrm{H}, \mathrm{H}_{2^{\prime}}, \mathrm{dd}\right), 6.6 \mathrm{ppm}$ $(1 \mathrm{H}, \mathrm{m}), 7.3(2 \mathrm{H}, \mathrm{m})$; ES-MS $m / z 978.8(\mathrm{M}+\mathrm{H})^{+}$; mp: $166^{\circ} \mathrm{C}$.

\section{Iron Complex 3e}

$343 \mathrm{mg}(0.35 \mathrm{mmol})$ of 2'-O-(3,4-dihydroxybenzoyl)spiramycin 3d was dissolved in $10 \mathrm{ml}$ anhydrous $\mathrm{MeOH}$, $43 \mathrm{mg}(0.12 \mathrm{mmol}) \mathrm{Fe}(\mathrm{acac})_{3}$ and $0.1 \mathrm{ml}$ of triethylamine. The reaction mixture was stirred 3 hours at room temperature. The crude product was filtered on Sephadex LH-20 to yield $230 \mathrm{mg}$ of complex 3e (33\%). $\mathrm{C}_{150} \mathrm{H}_{228} \mathrm{~N}_{6} \mathrm{O}_{51} \mathrm{Fe}$, MW 2987.34; ES-MS $\mathrm{m} / \mathrm{z} 2984$ 
$(\mathrm{M}+\mathrm{H})^{+\cdot}$ (for Mcalc 2985.48); UV: 3e $\lambda_{\max }(\mathrm{MeOH}) \mathrm{nm}$ 290, 537 (Fe-O), 3d $\lambda_{\max }(\mathrm{MeOH}) \mathrm{nm} 282,290 ; \mathrm{Fe}(\mathrm{acac})_{3}$ $\mathrm{nm} \mathrm{279,} 430(\mathrm{Fe}-\mathrm{O})$; since iron(III) is paramagnetic the analogous $\mathrm{Ga}(\mathrm{III})$ complex $3 \mathrm{f}$ was synthetized using $\mathrm{Ga}\left(\mathrm{NO}_{3}\right)_{3}$.

${ }^{1} \mathrm{H}$ NMR $\left(400 \mathrm{MHz}, \mathrm{CDCl}_{3}\right) \delta 2.9\left(1 \mathrm{H}, \mathrm{H}_{4}{ }^{\prime \prime}, \mathrm{d}\right), 4.9$ (1H, $\mathrm{H}_{2}$, dd), 6.44 (1H, d), $7.11(1 \mathrm{H}, \mathrm{s}), 7.16(1 \mathrm{H}, \mathrm{d})$.

\section{2'-O-(2,3-Dihydroxybenzoyl)spiramycin $\mathbf{3 g}$}

The same procedure as for $\mathbf{3 d}$ but using CDI instead of DCC yielded $10 \%$ of the title compound. $\mathrm{C}_{50} \mathrm{H}_{78} \mathrm{~N}_{2} \mathrm{O}_{17}$, MW 979.18; ${ }^{1} \mathrm{H}$ NMR $\left(400 \mathrm{MHz}, \mathrm{CDCl}_{3}\right) \delta 2.9(1 \mathrm{H}$, $\left.\mathrm{H}_{4^{\prime \prime}}, \mathrm{d}\right), 5.1\left(1 \mathrm{H}, \mathrm{H}_{2^{\prime}}\right.$, dd), $6.7(1 \mathrm{H}, \mathrm{m}), 7.2(2 \mathrm{H}, \mathrm{m})$; ES-MS $m / z 978.6(\mathrm{M}+\mathrm{H})^{+\cdot}$.

Anal Calcd for $\mathrm{C}_{50} \mathrm{H}_{78} \mathrm{~N}_{2} \mathrm{O}_{17}$ : C 61.33, H 8.03, N 2.86. Found:

C 60.81, H 7.97, N 4.48 .

$2^{\prime}-O-[4-N-(2,3-$ Diacetox ybenzoyl $)]$ butanoylspiramycin $\mathbf{3 h}$

The same procedure as for $3 \mathbf{c}$ but using CDI instead of DCC yielded $27 \%$ of the desired compound $\mathbf{3 h}$. $\mathrm{C}_{58} \mathrm{H}_{89} \mathrm{~N}_{3} \mathrm{O}_{20}$, MW 1148.36; ${ }^{1} \mathrm{H}$ NMR $(400 \mathrm{MHz}$, $\left.\mathrm{CDCl}_{3}\right) \delta 2.9\left(1 \mathrm{H}, \mathrm{H}_{4^{\prime}}, \mathrm{d}\right), 4.9\left(1 \mathrm{H}, \mathrm{H}_{2^{\prime}}, \mathrm{dd}\right), 6.85(1 \mathrm{H}$, m), $7.2(2 \mathrm{H}, \mathrm{m})$; ES-MS $m / z 1147.80(\mathrm{M}+\mathrm{H})^{+\cdot}$.

Anal Calcd for $\mathrm{C}_{58} \mathrm{H}_{89} \mathrm{~N}_{3} \mathrm{O}_{20}$ : C 60.66, H 7.81, N 3.66. Found:

C $58.60, \mathrm{H} 7.64, \mathrm{~N} 3.32$.

$2^{\prime}-O-[N-(2,3-$ Diacetoxybenzoyl)-(L)-alanyl $]$ spiramycin $3 \mathbf{i}$

The same procedure as for $3 \mathrm{~h}$ yielded $24 \%$ of compound 3i. $\mathrm{C}_{57} \mathrm{H}_{87} \mathrm{~N}_{3} \mathrm{O}_{20}$, MW 1134.34; ${ }^{1} \mathrm{H}$ NMR $\left(400 \mathrm{MHz} \mathrm{CDCl}_{3}\right) \delta 2.9\left(1 \mathrm{H}, \mathrm{H}_{4^{\prime \prime}}, \mathrm{d}\right), 4.9\left(1 \mathrm{H}, \mathrm{H}_{2^{\prime}}, \mathrm{dd}\right)$, $6.8 \sim 7.3(3 \mathrm{H}, \mathrm{m})$.

$\begin{array}{ll}\text { Anal Calcd for } \mathrm{C}_{57} \mathrm{H}_{87} \mathrm{~N}_{3} \mathrm{O}_{20} \text { : } & \text { C 60.36, H 7.73, N 3.70. } \\ \begin{array}{ll}\text { Found: } & \text { C 56.71, H 7.33, N 3.56. }\end{array}\end{array}$

\section{Neospiramycin 2}

$1 \mathrm{~g}(1.19 \mathrm{mmol})$ of spiramycin was dissolved in $20 \mathrm{ml}$ of $\mathrm{HCl} 1 \mathrm{~N}$. The reaction mixture was stirred 8 hours at $40^{\circ} \mathrm{C}$. The $\mathrm{pH}$ was ajusted to $9 \sim 10$ and the product was extracted by $\mathrm{CH}_{2} \mathrm{Cl}_{2}$. The solvent was evaporated and the crude mixture was chromatographed by MPLC on reversed phase LiChroprep RP-18 (Merck) with $\mathrm{H}_{2} \mathrm{O}$ (citric acid $\mathrm{pH}=3,450 \mathrm{mg} / \mathrm{liter}$ ) $74 \% / \mathrm{CH}_{3} \mathrm{CN} 26 \%$ as elution system. After extraction $270 \mathrm{mg}$ of neospiramycin 2 were obtained (33\%). $\mathrm{C}_{36} \mathrm{H}_{62} \mathrm{~N}_{2} \mathrm{O}_{11}$, MW 698.43; in ${ }^{1} \mathrm{H}$ NMR, the $4 "$ proton signal at $\delta=2.9 \mathrm{ppm}$ and the $1^{\prime \prime}$ proton signal at $\delta=5.0 \mathrm{ppm}$ disappeared, 2.2 and 2.5 $\mathrm{N}\left(\mathrm{CH}_{3}\right) ;$ ES-MS $m / z \quad 698.6(\mathrm{M}+\mathrm{H})^{+}{ }^{+}, \quad m / z \quad 557.3$ $(\mathrm{M} \text { - forosamine })^{+}$.
Anal Calcd for $\mathrm{C}_{36} \mathrm{H}_{62} \mathrm{~N}_{2} \mathrm{O}_{11}$ : C 61.87, $\mathrm{H}$ 8.94, N 4.01. Found:

C 59.19, H 8.59, N 3.12 .

$2^{\prime}, 4^{\prime}$-Di-O-[4- $N$-(2,3-diacetoxybenzoyl)butanoyl]neospiramycin $3 \mathbf{j}$

$549 \mathrm{mg}$ ( $1.70 \mathrm{mmol} ; 2.4$ equiv) of compound $10 \mathrm{a}$ were stirred in $20 \mathrm{ml} \mathrm{CH}_{2} \mathrm{Cl}_{2}$. After addition of $278 \mathrm{mg}$ ( $1.70 \mathrm{mmol}$; 2.4 equiv) of CDI the reaction mixture was stirred at room temperature for 1 hour. $495 \mathrm{mg}(0.71$ mmol) of neospiramycin in $20 \mathrm{ml} \mathrm{de} \mathrm{CH}_{2} \mathrm{Cl}_{2}$ were added dropwise and the reaction mixture was heated under reflux for 8 hours. The solvent was evaporated and the crude product was chromatographed by MPLC on reversed phase LiChroprep RP-18 (Merck) with $\mathrm{H}_{2} \mathrm{O}$ (citric acid $\mathrm{pH}=3,450 \mathrm{mg} / \mathrm{liter}$ ) $74 \% / \mathrm{CH}_{3} \mathrm{CN} 26 \%$ as elution system. After extraction $67 \mathrm{mg}$ of product $3 \mathrm{j}$ were obtained (7\%). $\mathrm{C}_{66} \mathrm{H}_{92} \mathrm{~N}_{4} \mathrm{O}_{21}$, MW 1309.48; ${ }^{1} \mathrm{H}$ NMR $\left(400 \mathrm{MHz}, \mathrm{CDCl}_{3}\right) \delta 6.8 \sim 7.8(6 \mathrm{H}, \mathrm{m})$; ES-MS $\mathrm{m} / \mathrm{z}$ $1310.77(\mathrm{M}+\mathrm{H})^{+}$.

\section{Acknowledgments}

The authors wish to thank J.-M. PARIS and J.-F. DesnotTeS for fruitful discussions and helpful advice.

H. Poras and G. KunEsCH thank the CNRS and Rhône-Poulenc-Rorer for the attribution of a grant and generous financial help.

\section{References}

1) Conen, M. L.: Antimicrobial resistance: Progress for Public Health. Trends in Microbiology 2: 422 425, 1994

2) Wooldridge, K. G. \& P. H. Williams: Iron uptake mechanisms of pathogenic bacteria. F.E.M.S. Microbiol. Rev. 12: 325 348, 1993

3) Miller, M. J. \& F. Malouin: The Development of Iron Chelators for Clinical Use. Ed., B. J. BERGERON, pp. 275 306, CRC Press, Boca Raton, FL, U.S.A., 1994

4) Bickel, H.; E. Gaumann, G. Nussberger, P. Reusser, E. Vischer, W. Vosser, A. Wettstein \& H. ZÄhNer: Über die Isolierung und Charakterisierung der Ferrimycine $A_{1}$ und $A_{2}$, neuer Antibiotika der SideromycinGruppe. Helv. Chim. Acta 43: 2105 2118, 1960

5) Benz, G.; T. Schroder, J. Kurz, C. Wunsche, W. Karl, G. Steffens, J. Pfitzner \& D. Schmidt: Konstitution der Desferriform der Albomycine $\delta_{1}, \delta_{2}, \varepsilon$. Angew. Chem. Suppl. 1322 1335, 1982

6) Bricard, L.; F. Ramiandrasoa, G. Kunesch, A. Gaudemer, C. Loubeyre \& A. ANDremont: French Patent $\mathrm{N}^{\circ}$ 9405792, 1994

7) Pinnert-Sindico, S.; L. Ninet, J. Preud'homme \& C. Cosar: Antibiotics Annual, pp. 724 725, 1954 1955

8) Omura, S.: Macrolide Antibiotics: Chemistry, Biology and Practice, Academic Press, Orlando, FL, U.S.A., 1984

9) Kunesch, N.; C. Miet \& J. Poisson: Utilisation de la guanidine comme agent désacétylant sélectif: Une méthode de désacétylation instantanée applicable aux sucres. Tetrahedron Lett. 28: 3569 3572, 1987 
10) Reissbrodt, R.; L. Heinisch, U. Mollmann, W. Rasch \& H. UlBRICHT: Growth promotion of synthetic catecholate derivatives on Gram-negative bacteria. Biometals 6: 155 162, 1993

11) Husson, A.; R. Besselievre \& H.-P. Husson: A short and efficient synthesis of phenolcarboxamides. Tetrahedron Lett. 24: $1031 \sim 1034,1983$

12) Ramu, K.; S. Shringarpure, S. Cooperwood, J. M. Beale \& J. S. Williamson: ${ }^{1} \mathrm{H}-\mathrm{NMR}$ and ${ }^{13} \mathrm{C}-\mathrm{NMR}$ spectral assignments of spiramycins I and III. Pharmaceutical Research 11: 458 465, 1994

13) Sano, H.; H. Tanaka, K. Yamashita, R. Okachi \& S. OMURA: Synthesis and in vitro and in vivo activities of $3^{\prime \prime}, 4^{\prime \prime}$-diacylates and $3,3^{\prime \prime}, 4^{\prime \prime}$-triacylates of spiramycin I. J. Antibiotics 37: $760 \sim 772,1984$

14) SCriven, E. F. V.: 4-Dialkylaminopyridines: Super Acylation and Alkylation Catalysts. Chem. Soc. Rev. 12: $129 \sim 161,1983$ 